\title{
Determination of Top-Quark Properties
}

\author{
Yuji Yamazaki \\ on behalf of ATLAS, CMS and LHCb Collaborations \\ Graduate School of Science, Kobe University, \\ Kobe, Hyogo, 657-8501 Japan \\ *E-mail: yamazaki@phys.sci.kobe-u.ac.jp
}

\begin{abstract}
Top quarks are copiously produced at the LHC. The measurements are in stage for precision. A review on top-quark properties measured by the ATLAS, CMS and LHCb collaborations is presented, including the mass of the top quark, coupling, production cross sections and decay properties.
\end{abstract}

Keywords: top quark, electroweak, QCD, Standard Model, BSM Physics

\section{Introduction}

The discovery of top quarks in 1994 has opened up completely new possibilities of testing the Standard Model (SM). The top quark has large coupling to the Higgs boson and has been having large sensitivity to SM and Beyond-Standard-Model (BSM) physics by looking at top quark radiative corrections in SM electroweak measurements. After the discovery of the Higgs boson, the top quark properties became even more important since it provides unique opportunities for consistency checks between $m_{W}, m_{\text {top }}, m_{H}$ and other EW measurements. Deviation among these masses is an implication for presence of physics beyond the Standard Model. In addition, couplings, spin properties and high- $p_{T}$ cross section behaviour are the important probe for new physics strongly coupled to the top quark at TeV scale.

A top quark pair, $t \bar{t}$, is mostly produced through gluon-gluon fusion diagrams at the LHC energies $(7-13 \mathrm{TeV})$. The production cross section $(\simeq 820 \mathrm{pb})$ is much larger at the LHC than that at the Tevatron: cross section increases rapidly with $\sqrt{s}$. This comes mainly from parton densities, which also increases rapidly with $1 / x$, where $x$ here represents the longitudinal momentum fraction of the parton in the proton. The large production rate allows to investigate the properties of the top quarks with very high precision. More than 40 millions of the $t \bar{t}$ pairs had already been produced for each experiment at the time of this symposium.

So-called "single-top" production cross sections are also very large, where a top quark is produced from a bottom quark through flavour excitation by a $W$ boson. The single-top cross sections amount to about $1 / 4$ of the pair production. Therefore, the singly-produced top quarks are not only sensitive to its coupling to weak bosons but also give another possibility to measure top quark properties.

The decay of the top quarks is in two steps. First it decays to a bottom quark and a $W$ boson, $t \rightarrow b+W$, for almost $100 \%$ because of the $\left|V_{t b}\right|$ element in the CKM matrix being very close to unity. Since it is a weak decay, the helicity of 
the top quark is trasnferred to $W$. One very important feature of this decay is that the lifetime is very short $(\Gamma \simeq 1.3 \mathrm{GeV})$, unlike other heavy quark decays, because of the large $Q$ value. This makes the top quark very unique: it decays before hadronisation i.e. the decay occurs as a bare quark and produces a bottom quark before hadronisation. The top decay, therefore, is expected to be free from soft QCD effects.

The top decay can further be classified by the second step, the decay of the $W$ boson: semi-leptonic decay $\left(t \rightarrow \ell \nu_{\ell} b\right)$ and hadronic decay $(t \rightarrow q \bar{q} b)$. Three combinations are possible In case of the top pair production. They are called dilepton (both semi-leptonic), $\ell+$ jet (semi-leptonic and hadronic) and all-hadronic (both hadronic) channels. The $\ell+$ jet channel has one neutrinos while dilepton decay contains two neutrinos from the top decay. The advantage of the hadronic decay is absence of neutrinos while the leptons are much more precisely reconstructed in other channels. Repeating measurements in the three decay patterns is often beneficial because of different experimental systematics.

The top quarks, especially those from the pair production, are quite pure source of the bottom quarks. The background contribution is practically negligible for dilepton channel. The jets in these events are a good source of bottom quarks. This fact is used for calibrating detectors, understanding $b$-tagging algorithms and jet energy scale of the $b$-jets and other purposes.

This article reviews recent measurements on top properties, such as top mass, top production cross sections, top couplings and single-top production, with an emphasis on new results from the $\mathrm{ATLAS}^{1}, \mathrm{CMS}^{2}$ and $\mathrm{LHCb}^{3}$ collaborations.

\section{Top Quark Mass}

Since a top quark decays before hadronisation, one would assume that the invariant mass of the top decay products corresponds to the bare mass of the top quark with good precision, of order of $\Lambda_{\mathrm{QCD}} \simeq 200 \mathrm{MeV}$. Measurements of $m_{\text {top }}$ through the reconstruction of the top decay products is called "direct method". Experimentally, the top quarks mass could in principle be reconstructed by using hadronic decay, where all the decay products are visible. For the semi-leptonic decay, the invariant mass of a part of the decay product, such as the invariant mass $M_{\ell b}$ of the $b$-jet and the charged lepton $\ell$, still strongly reflects assumed $m_{\text {top }}$ values.

Figure 1 shows the current status of top quark measurement values and their uncertainties from the Tevatron and the LHC experiments. The precision from the LHC experiments now surpasses those from Tevatron. The combined result from the ATLAS experiment is that $m_{\text {top }}=172.84 \pm 0.70 \mathrm{GeV}$, where the uncertainty is decomposed into \pm 0.34 (stat.) \pm 0.67 (syst.): the statistical uncertainty is not the dominant source. The best single measurement is from $\mathrm{CMS}^{4}, m_{\text {top }}=172.35 \pm$ $0.51( \pm 0.36 \pm 0.67)$, using the $\ell+$ jet channel. The CMS combined results is with the uncertainty of $0.48 \mathrm{GeV}$, which corresponds to $0.28 \%$ precision.

The best measurement from CMS make use of the kinematic fit ${ }^{6}$. For each event, 


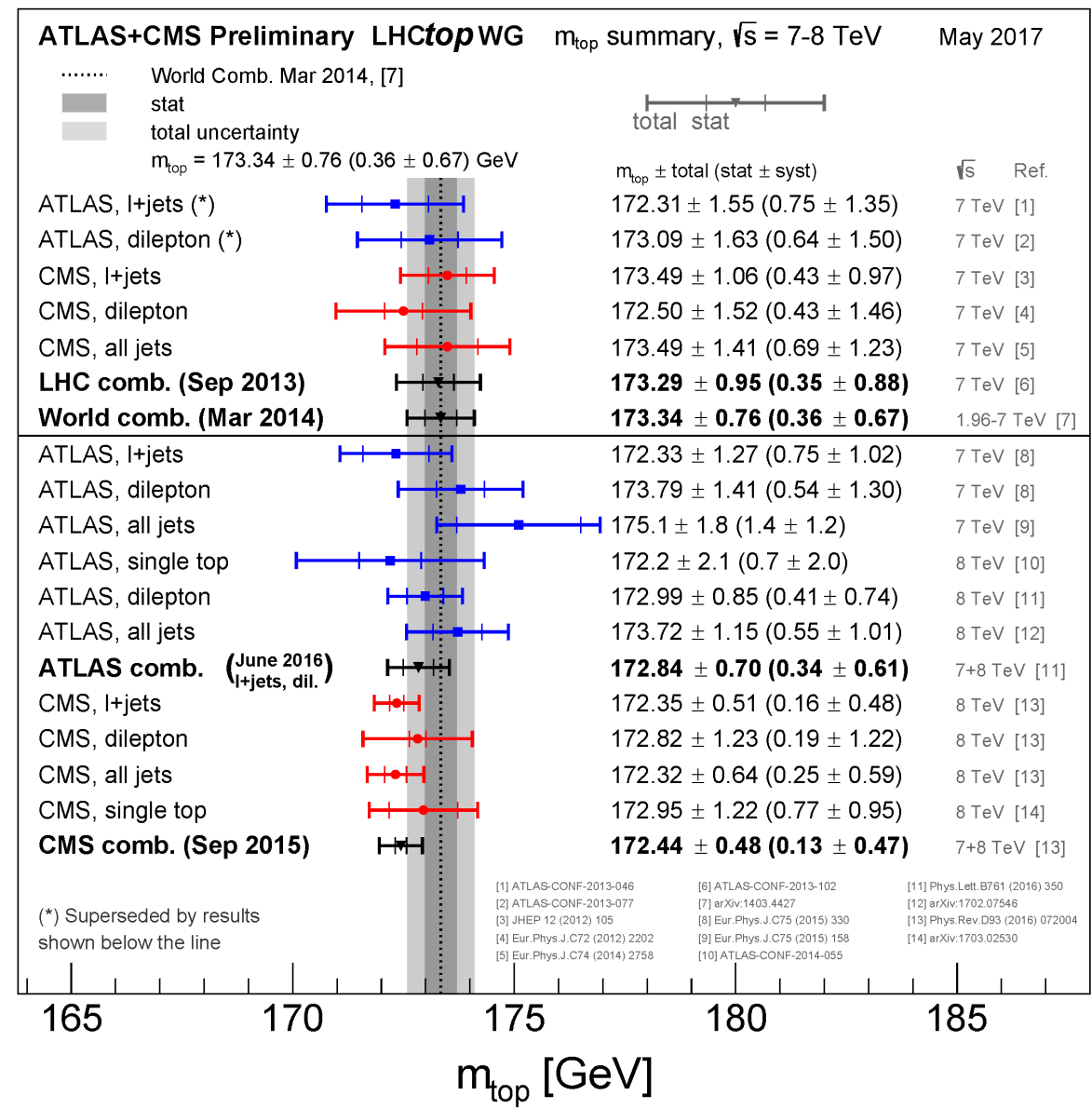

Fig. 1. Summary of the ATLAS and CMS direct $m_{\text {top }}$ measurements, in comparison to the LHC and Tevatron+LHC combinations. The figure was prepared by LHC Top Working Group ${ }^{5}$.

a likelihood is calculated for assumed $m_{\text {top }}$ and values of kinematical variables. The $m_{\text {top }}$ value is determined by maximising the product of the likelihood for all the events, scanning over assumed $m_{\text {top }}$ values. In order to further reduce the experimental uncertainties, the likelihood is also combined with the likelihood for the jet energy scale (JES) determined from the $W$ mass spectra in the hadronic decays of the top quarks, or the JES scale parameter extracted from other events containing hadronic decays of $W$ 's is included. An example of the reconstructed mass spectrum is shown in Fig. 2(left).

The benefit of using kinematic fit is demonstrated in Fig. 2(right), where the reconstructed mass as a function of $p_{T}$ of hadronically decayed top quarks is shown. No strong dependence of the mass on $p_{T}$ is observed both in the data and various 
models simulating the top quark production. This reduces the model dependence, especially on the distributions of kinematic variables in the models.
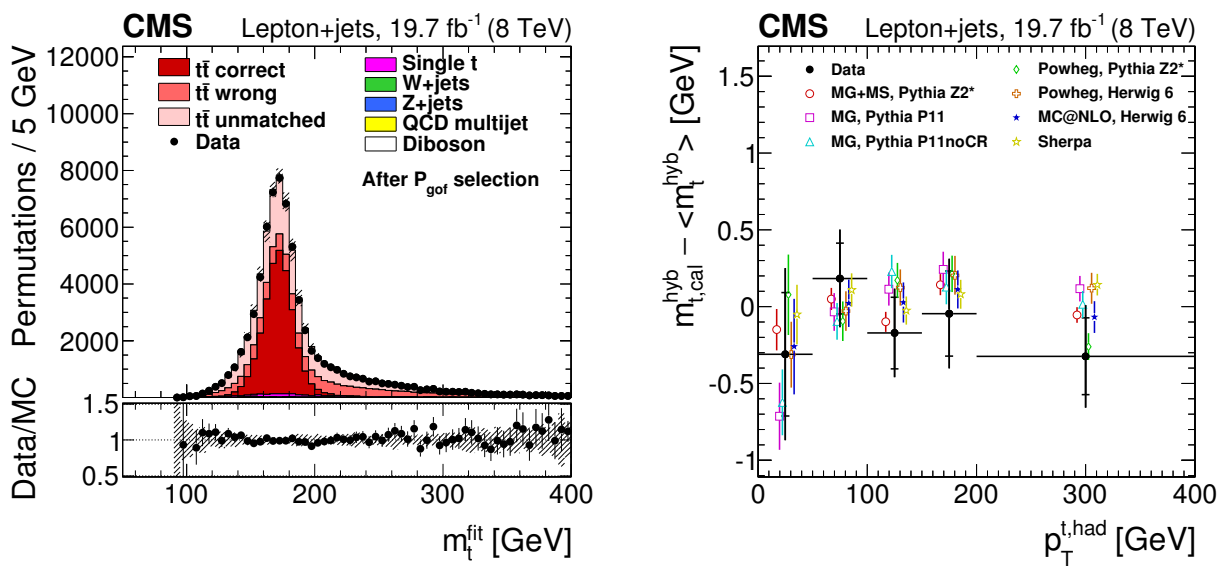

Fig. 2. An example of the performance in the $m_{\text {top }}$ measurement using $\ell+$ jet channel by $\mathrm{CMS}^{4}$. (left) Mass spectrum of the hadronically decayed top quark candidates after selecting events with good likelihood in kinematic fit (right) dependence of reconstructed $m_{\text {top }}$ on the top quark $p_{T}$.

The invariant mass of the end product of the decay, however, does not exactly correspond to the quark mass of the top quark. For lighter quarks, e.g, a $b$-quark, the mass of a $B$ meson is considered to be significantly heavier than the bare mass of the heavy quark $\left(m_{B_{0}}-m_{b}>\Lambda_{\mathrm{QCD}}\right)$. This effect should be smaller for the top quark but may still be important. Moreover, several issues arise when comparing the direct mass and the bare quark mass, as illustrated in Fig. 3. First is that jets are used instead of the decay partons when measuring the momenta. A significant part of the energy of the corresponding parton leaks out of the jets through final-state QCD radiation, in particular if the jet area is chosen to be small, while a sizeable amount of particles enters into the jets through soft effects (initial state QCD radiation, particles from multi-parton interactions etc.), especially in case the jet area is large. In addition, since the decay life time is short, the partons after the decay may still interact with other partons i.e. some colour reconnection occurs, which may change the way for partons to fragment. These points are specific problem for the top quarks since the partons after the decay remains coloured objects. Furthermore, the life time of top quark between the production and decay is so short that the top quark may be off-shell, distorting resonance mass shape.

Many of these effects, which may lead to bias in reconstructed mass peak of the top decay products, are simulated differently in every event generator. This means that the difference between the bare top quark mass and the reconstructed mass is strongly model-dependent. In fact, for many of the direct mass measurements, one of the dominant source of systematic uncertainties is model dependence, such as the matrix element simulation, parton shower simulation or matching between the 


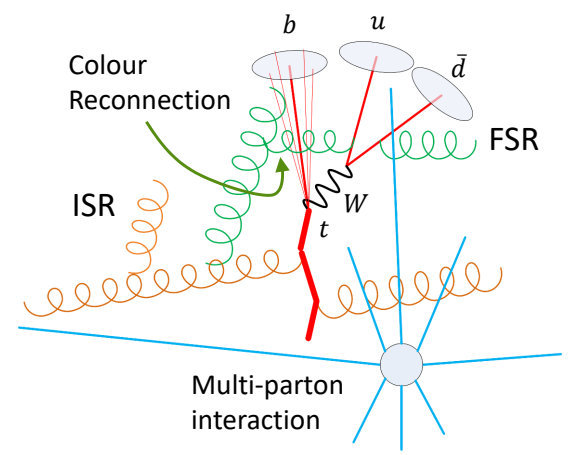

Fig. 3. An illustration on various effects affecting the top mass reconstructed from the decay particles.

matrix element calculation and the fragmentation model. They often have similar magnitudes to the experimental uncertainties, e.g. jet energy scale, $b$-jet energy scale or background estimation. It is, therefore, desired to cross-check the method of direct mass reconstruction using alternative methods.

Many of such alternatives use leptons to calculate partial masses, even though the mass reconstructed from kinematical variables from leptons can avoid only the theoretical uncertainties related to jets. One example is to utilise leptonic decays of $J / \psi^{7}$, which is originated from the $b$-quark from the top decay. The invariant mass of $J / \psi$ and the charged lepton from the decay of $W$ is compared to templates of various $m_{\text {top }}$ values. Although the peak of the three-lepton mass is much smaller than $m_{\text {top }}$ since it misses the neutrino from the $W$ decay as well as the fragmented light hadrons from the $b$-quark. The peak of the three-lepton mass is modelled with small theoretical uncertainty. In fact, the uncertainty on the top mass determination from $b$-quark fragmentation $(0.37 \mathrm{GeV})$, governing the fraction of the $b$-quark momentum to $J / \psi$, is much smaller than the largest uncertainty, which is top $p_{T}$ modelling $(0.64 \mathrm{GeV})$. Although this measurement is statistically not competitive for the moment, the method would have potential to become one of the most precise measurement with larger integrated luminosity, given the small theoretical uncertainties.

Such alternative methods are still not free from uncertainties related to internal lines in the top production diagram such as colour reconnection or off-shell effect in the top quark decay. The production cross section or differential cross sections, on the other hand, are much less affected by these effects. The mass of the top quark can be obtained by using the measured cross sections, since the cross section reflects the mass in the propagator. The mass obtained through cross section behaviour is 
called "pole mass".

The CMS collaboration has determined the top mass by comparing the total pair production cross section with NNLO+NNLL calculation ${ }^{9}$, obtaining $m_{\text {top }}=$ $173.8_{-1.8}^{+1.7} \mathrm{GeV}$. Another example is to use kinematical distributions of leptons in $e \mu$ dilepton channel of the top-pair production ${ }^{8}$, such as $p_{T}^{\ell}, p_{T}^{e \mu}, m^{e \mu}$ etc. Figure 4 (left) shows an example of how such kinematical spectrum changes in shape according to input $m_{\mathrm{top}}$. It is shown that the $p_{T}^{e \mu}$ gives smaller tail with decreasing top mass. By using seven other variables and performing combined fit, a $m_{\text {top }}$ value is obtained with decent precision: $m_{\text {top }}=173.2 \pm 0.9$ (stat.) \pm 0.8 (exp.) \pm 1.2 (theo.). The uncertainty is dominated by renormalisation and factorisation scales; soft QCD uncertainties are suppressed.
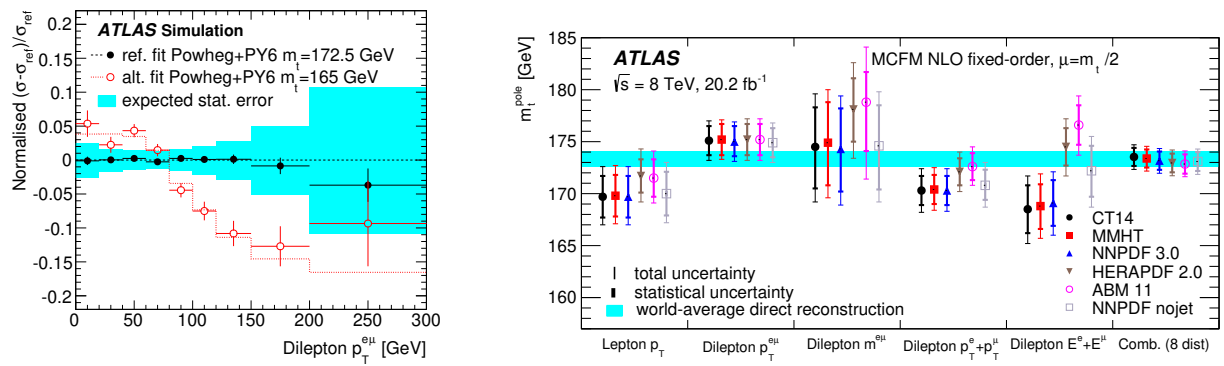

Fig. 4. Top mass determination by ATLAS using dilepton kiematics ${ }^{8}$. (left) Relative deviations in cross sections $\left(\sigma-\sigma_{\text {ref }}\right) / \sigma$ from the reference cross-section values as a function of dilepton $p_{T}^{e \mu}$, when $m_{\text {top }}=172.5 \mathrm{GeV}$ is changed to $165 \mathrm{GeV}$. (right) measurement of $m_{\text {top }}$ using lepton kinematic distributions in comparison to MCFM prediction with various PDF sets.

In these measurements reviewed here, there are three common major sources of systematic uncertainties: the jet and $b$-jet energy scale, modelling of top kinematics and decay, and cross section prediction such as scale uncertainties in QCD calculations. While the first one is experimental, the latter two are related to theory, which could be improved by giving feedback from measurements. It should, therefore, be possible to improve the mass measurement further from current precision $(\simeq 0.5 \mathrm{GeV})$ significantly.

\section{Differential cross sections}

Cross section measurements are primary mean to understand the theoretical modelling on production and decay mechanism. The measurement should be as differential as possible so that the models can be tested in each small area of the phase space. The observables should also have good correlation to the measured quantities on the detector level in order to minimise the uncertainties arising from extrapolation from the detector to the truth level. For that reason, the top quarks in cross section measurements are defined as "pseudo-top" quarks using variables 
related to their decay products after hadronisation, such as momentum and flavour of particle-level (truth hadron) jets. In addition, the variables to be used in measurements should be "well-defined" so that the observables on particle level have good correlation to the partonic variables.

Thus obtained particle-level cross sections can directly be compared to various generator models. Depending on the variables used, the differential cross sections would be sensitive to various aspects of models, such as matrix element, parton shower, hadronisation or parton-density functions (PDFs) used in the simulation. Once the behaviour of the cross sections with respect to the models is understood, the systematic errors due to modelling can be reduced by choosing models describing the data better or tuning the generator models.

The differential cross sections, in particular at high- $p_{T}$, are also sensitive to BSM physics by comparing with SM predictions. Top quarks are also important source of background in many BSM searches because of their complicated final state with leptons, jets, $b$-jets and missing $E_{T}$. Understanding normalisation of the top quark cross sections as well as the behaviour of events at the edge of phase space would help in reducing the uncertainties in background, and thus increasing the sensitivity on BSM searches.

The hadronically decayed top quarks at high $p_{T}, p_{T}^{\text {top }} \gg m_{\text {top }}$, has three jets in collimated $\eta-\phi$ space, while at $p_{T}^{\text {top }} \lesssim m_{\text {top }}$ three jets are well separated. For such collimated decay, the entire objects from a top quark decay may be contained in a single cone. The momentum of the top quark then can be obtained from largeradius jets, e.g. the jet radius $R=1.0$. Such "fat jets" can be identified as a top quark decay product if clear structure of three jets is observed, one of them being $b$-tagged, other two being within a window of $M_{W}$ etc. This technique is called boosted-top tagging, which is widely used to improve the tagging efficiency at high- $p_{T}$, complimenting top tagging that requests three well-separated jets (called "resolved top"). An example of measurements using boosted tagging at $13 \mathrm{TeV}$ LHC run with $14.7 \mathrm{fb}^{-1}$ of the data ${ }^{10}$ is shown in Fig. 5 . The highest $p_{T}^{\text {top }}$ is now beyond $1 \mathrm{TeV}$, or above $2 \mathrm{TeV}$ in $m_{t \bar{t}}$.

Large statistics from $13 \mathrm{TeV}$ collisions also allow to have high precision measurements in other channels. In order to avoid theoretical uncertainties, cross sections are measured as a function of kinematical variables of decay objects, such as $p_{T}^{\ell}$, or event kinematics like $H_{T}$, instead of properties related to top quark momentum. Figure 6 shows results from CMS $\ell+$ jet measurement using full statistics of 2015 and 2016 data $^{11}$ in comparison to various models: different combination of matrix element (POWHEG, MadGraph5) and parton showers (PYTHIA8, HERWIG++) simulation, with different matching scale between the matrix element and parton shower and various parameters on parton shower suppression. The data are enough precise to discriminate models causing shifts of the order of $10 \%$.

Many of these models show a tendency that the $p_{T}^{\ell}$ spectrum is harder than the data, which is one of features common in many measurements. Figure 7 shows 

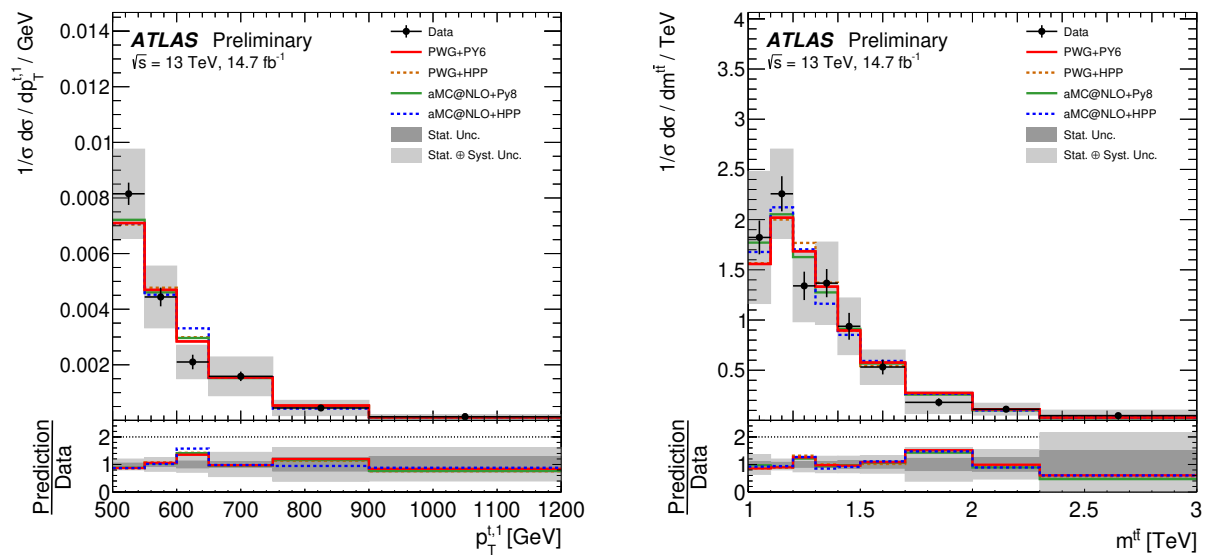

Fig. 5. Normalised top quark differential cross sections in fiducial phase-space measured by AT$\mathrm{LAS}^{10}$ as a function of (left) transverse momentum of the leading top-quark jet (right) invariant mass of the $t \bar{t}$ system. The top quarks are tagged by boosted top technique.
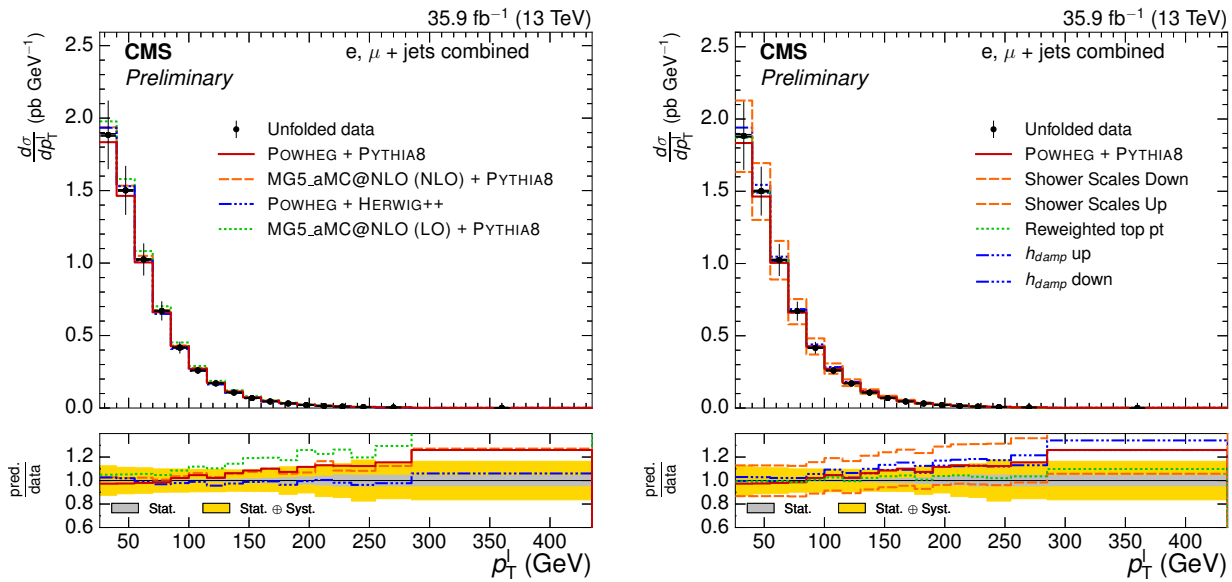

Fig. 6. Absolute differential cross sections as a function of $p_{T}^{\ell}$ using $\ell+$ jet channel of $13 \mathrm{TeV}$ data $^{11}$. (left) Comparison to models with different matrix element calculation and parton shower and (right) to models with various parton shower parameters.

examples from other cross section measurements vs. $p_{T}^{\text {top }}$ from ATLAS ${ }^{12,13}$ and $\mathrm{CMS}^{14,15}$. Many of the models predict harder spectrum than the data. This may imply that the models do not simulate some energies not given to the system of the top quark pair but to additional jets, either inside or the outside the fiducial acceptance of the detector $\left(\eta_{\text {jet }} \lesssim 2.5\right)$. In fact, recent calculation ${ }^{16}$ shows that the spectrum becomes softer by increasing the order of approximation from NLO to NNLO. Models reproducing such behaviour of the data, in particular event generators, are awaited for reducing the systematic effects due to models in precision 
measurements.
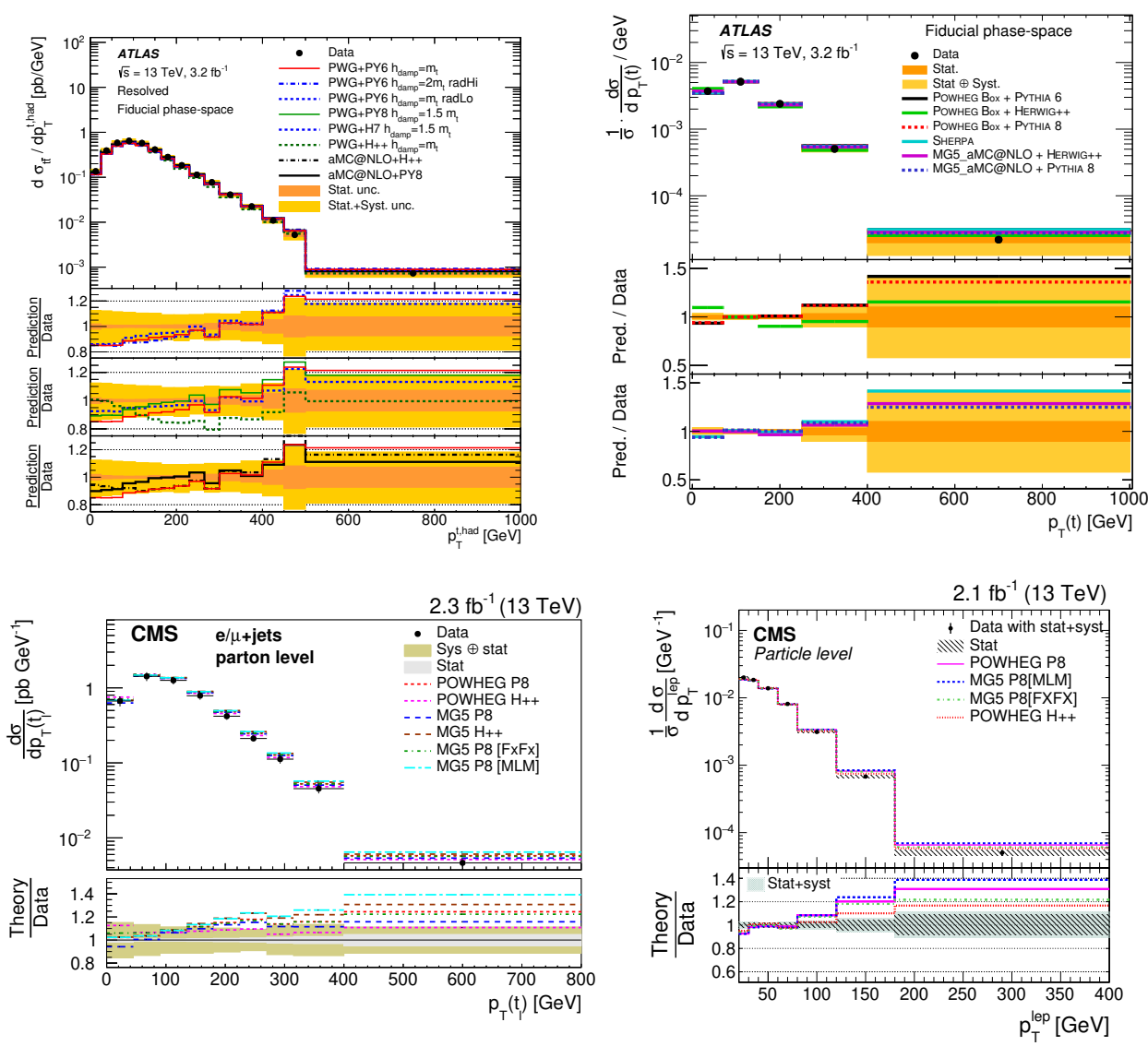

Fig. 7. Differential cross sections as a function of $p_{T}^{\text {top }}$ for (upper left) ATLAS $\ell+$ jet $^{12}$, (upper right) ATLAS dilepton ${ }^{13}$, (lower left) CMS $\ell+$ jet $^{14}$ and (lower right) CMS dilepton ${ }^{15}$ measurements using $13 \mathrm{TeV}$ collision data.

The differences between top production models are often more clearly seen in the properties related to additional QCD radiation, such as number of jets or $p_{T}$ imbalance between the two top quarks. Figure 8 shows the cross section for each number of jets (left) or additional jets to those originated from the top quark (right). $\mathrm{CMS}^{17}$ (Fig. 8(left)) used previous measurements at $8 \mathrm{TeV}$ to tune the parameters related to parton shower simulation in the model. The result of the tuned model is indicated as solid line in Fig. 8(left), giving very good description of the data. For ATLAS ${ }^{18}$ (Fig. 8(right)), the comparison is made with models not tuned to this particular data. The deviation between models show high sensitivity of the measurement.

The LHCb collaboration also have measured top production cross sections for the 

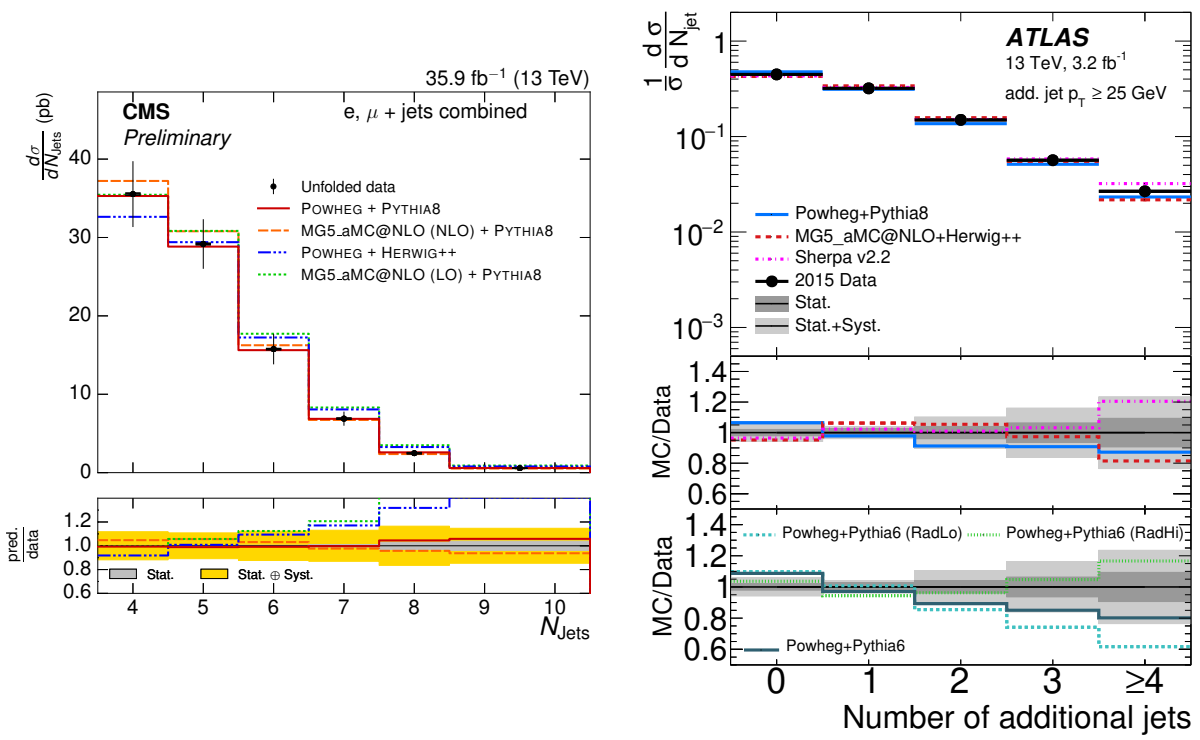

Fig. 8. Differential cross sections for top-pair production as a function of the number of jets from $\mathrm{CMS}^{17}$ (left) and the number of additional jets to the top quark jets from ATLAS ${ }^{18}$ (right).

first time ${ }^{19}$ with $4.9 \sigma$ significance. Since the $\mathrm{LHCb}$ detector covers forward rapidity region $(2<\eta \lesssim 4.5)$, the events are originated from parton-parton collisions with largely asymmetric configuration. The cross section, therefore, is sensitive to the parton densities of both high and low- $x$ gluons. The measured fiducial cross section, $0.05_{-0.01}^{+0.02}$ (stat.) $+0.02_{-0.01}$ (syst.) pb, is consistent with prediction of $0.045 \mathrm{pb}$.

\section{Top quark couplings and spin}

Although the top quark QCD coupling at around the scale of top mass is well tested through production cross section measurements, its electroweak couplings are not strongly constrained yet. Additional boson production with $t \bar{t}$ is sensitive to possible deviation of these couplings from the SM values. Top decay width is measured in order to find possible deviation in top couplings and couplings to unknown particle. The spin properties, such as spin correlation between the top and anti-top quarks in the $t \bar{t}$ production, the $V-A$ vertex structure of the top decay, can be measured by decay angles (not reviewed in this article).

The first observation on $t \bar{t} W$ and $t \bar{t} Z$ production by $\mathrm{CMS}^{20}$ with full 2015 and 2016 statistics at $13 \mathrm{TeV}$ is shown in Fig. 9. The $Z^{0}$ coupling to third-generation quarks is not experimentally well constrained. A good part of $Z^{0} \mathrm{~s}$ are radiated from the top quarks in the $t \bar{t} Z$ production, an example of which is shown in 9 (left). Such diagrams are sensitive to the top- $Z^{0}$ coupling and also important for understanding $t \bar{t} H$ process, since all the $t \bar{t} Z$ diagrams, especially those where a $Z^{0}$ is coupled to a top quark. has corresponding $t \bar{t} H$ diagrams. The cross sections are constrained 
at about $10 \%$ level (Fig. 9 (right)). The result is interpreted in terms of effective Lagrangian. Assuming that the coefficients to the effective terms are about unity, a presence of new physics through the effective operators in sub- $\mathrm{TeV}$ range is already excluded.
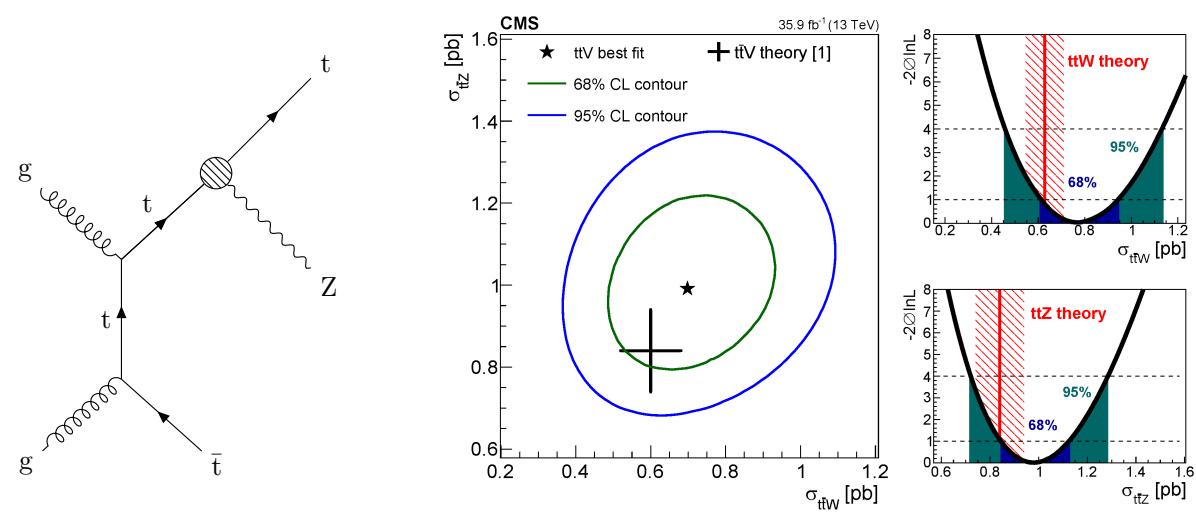

Fig. 9. (left) An example of LO diagram with top- $Z^{0}$ coupling through SM or new physics. (right) Result of simultaneous fit for $t \bar{t} W$ and $t \bar{t} Z$ cross sections ${ }^{20}$. The right panels show the individual cross sections along with 68 and $95 \%$ CL intervals.

The corresponding measurements with a photon in the final state, $t \bar{t} \gamma$, are performed by both ATLAS $^{21}$ and CMS $^{22}$ using full statistics of $8 \mathrm{TeV}$ data. The measurements show good agreement to the theoretical prediction. An example of kinematical distribution for signal and background is shown in Fig 10 (left). The differential cross section vs photon $p_{T}$ and $|\eta|$ are also both well reproduced by NLO prediction, as shown in Fig 10 (right).

The SM prediction on the top width is $1.322 \mathrm{GeV}$ (for $m_{\text {top }}=172.5 \mathrm{GeV}$ ). This is much smaller than experimentally reconstructed mass width due to detector resolution and QCD radiation effect as discussed in section 2. The tail of the distribution in reconstructed mass spectrum still shows shifts by changing the input top widths, as is shown in Fig. 11 (right). The most recent measurements show that top decay width is consistent with the SM prediction: $0.6<\Gamma_{t}<2.5 \mathrm{GeV}(95 \%$ CL) by $\mathrm{CMS}^{23}$ and $\Gamma=1.76 \pm 0.33$ (stat.) ${ }_{-0.68}^{+0.79}$ (syst.) by $\operatorname{ATLAS}^{24}$. These results indicate that the partial widths of top decay through non-SM particles, if any, is not much larger than that of the SM particles.

\section{Single-top production}

Single-top production is classified in three diagrams: $q b \rightarrow q^{\prime} t$ via $t$-channel $W$ boson exchange (called "t-channel"), $g b \rightarrow t W$ with top exchanged in $t$-channel $(W t)$, and the $s$-channel production where a $q \bar{q}^{\prime}$ state annihilates into a $W$, then goes to $t \bar{b}$ (s-channel). The $b$-quarks in the initial state is theoretically treated to be 

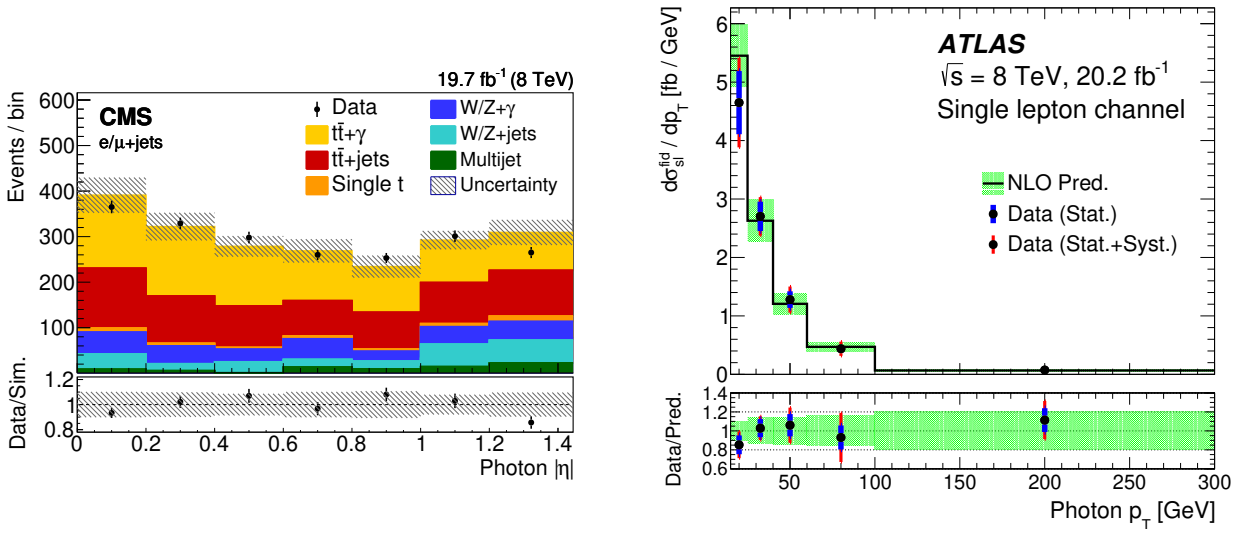

Fig. 10. (left)Photon $|\eta|$ distribution in $t \bar{t} \gamma$ event candidates with its decomposition to signal and various background sourcesCMS ${ }^{22}$. (right)Differential $t \bar{t} \gamma$ cross sections in photon $p_{T}{ }^{21}$.
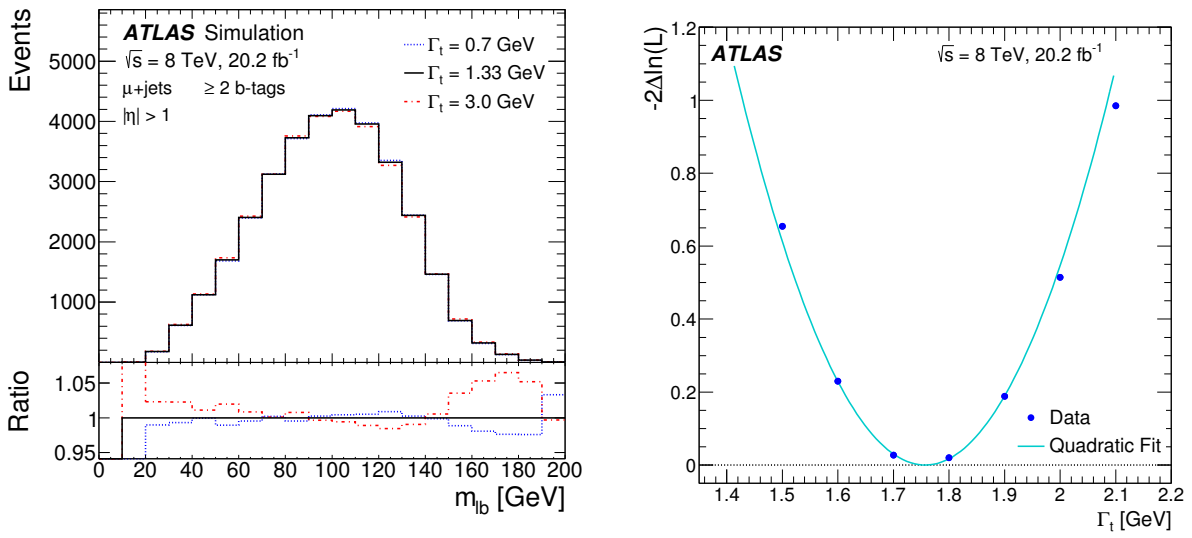

Fig. 11. Results from top width measurement by ATLAS ${ }^{24}$. (left) reconstructed $m_{l b}$, invariant mass of the $b$-jet and the lepton in the semi-leptonic decay simulated with different $\Gamma_{t}$ values. (right) Likelihood of the data as a function of the assumed $\Gamma_{t}$ in the templates.

either dynamically produced through gluon splitting (so-called four-flavour scheme) or directly originated from the incoming protons as an active flavour (five-flavour scheme).

The mass of the particles in the final state of the $t$-channel process is much lighter than that of $t \bar{t}$ production. This leads to a large cross section ( $\gtrsim 200 \mathrm{fb})$, as seen in Fig. 12. The background for $t$-channel can be well suppressed at the LHC, so that the single-top production can also be used for measuring top quark properties, in particular for top angular distribution studies, where the leptons from the semileptonic decays has been used. Only one neutrino is involved in semi-leptonic decays 
of for single-top production similarly to the $\ell+$ jet channel of pair production.

The observation on $s$-channel events suffers from large background since the production cross section is very small. The study at high energy collisions, i.e. 13 $\mathrm{TeV}$ instead of 7 or $8 \mathrm{TeV}$, remains to be difficult despite larger cross section, since the background rate increases proportionally.

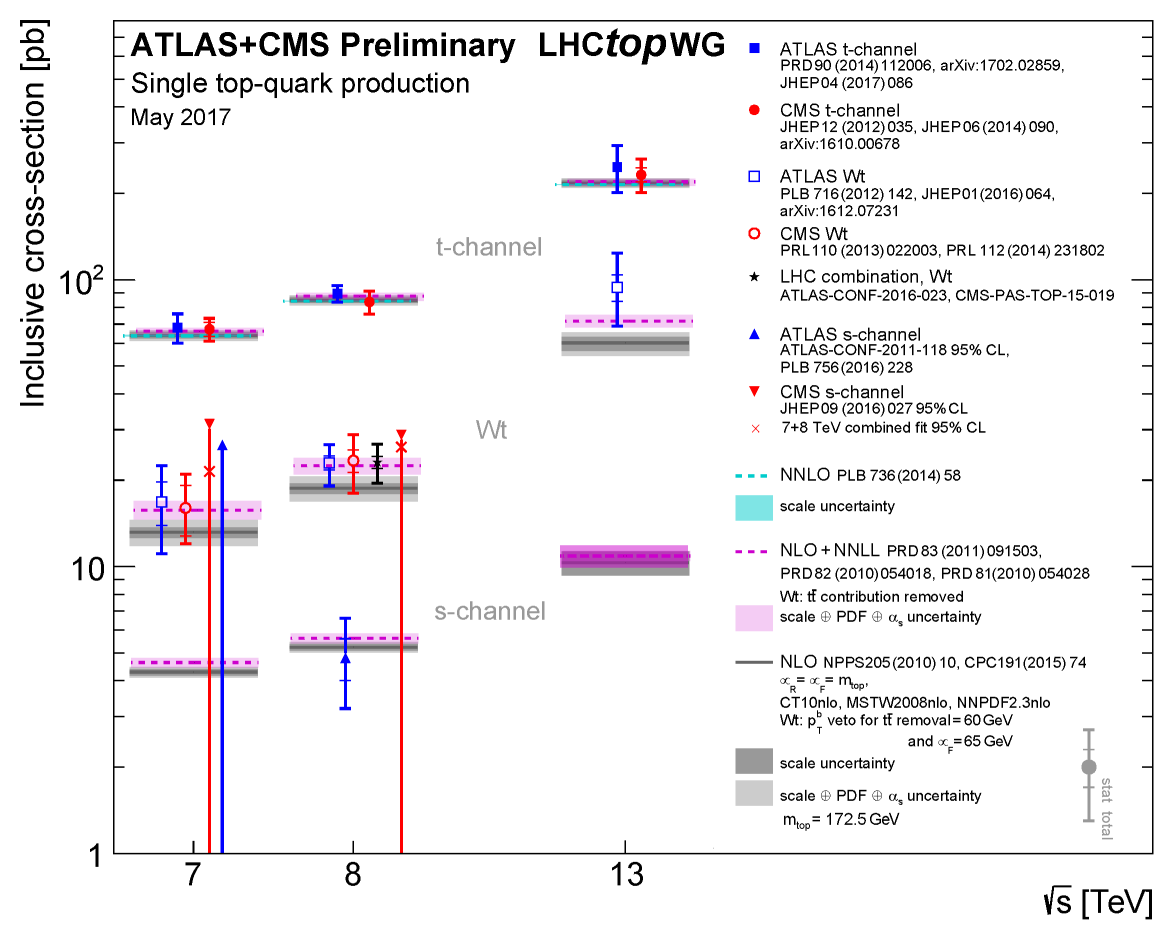

Fig. 12. Summary of ATLAS and CMS measurements of the single top production cross sections in various channels as a function of the centre-of-mass energy. The figure was prepared by LHC Top Working Group 25

The large number of events of $t$-channel production allows to measure differential cross sections for single-top production. Figure 13 (left) shows $p_{T}$ of the top quark from single-top production at $13 \mathrm{TeV}^{26}$. In this figure the prediction by the aMC@NLO model shows softer spectrum for the five-flavour scheme than the four-flavour scheme, demonstrating sensitivity to the choice of the flavour scheme. Figure 13(right) shows the $t q$ production cross section at $8 \mathrm{TeV}^{27}$ in comparison with models using four- or five-flavour schemes. Although there is a tendency that the five-flavour scheme predicts higher cross sections, both the theoretical and experimental uncertainties are much larger. Differential cross sections with higher statistics would help to disentangle various theoretical uncertainties, such as the scale uncertainty, PDF uncertainty and the flavour scheme.

Another example benefiting from high purity and abundant statistics in $t$ - 

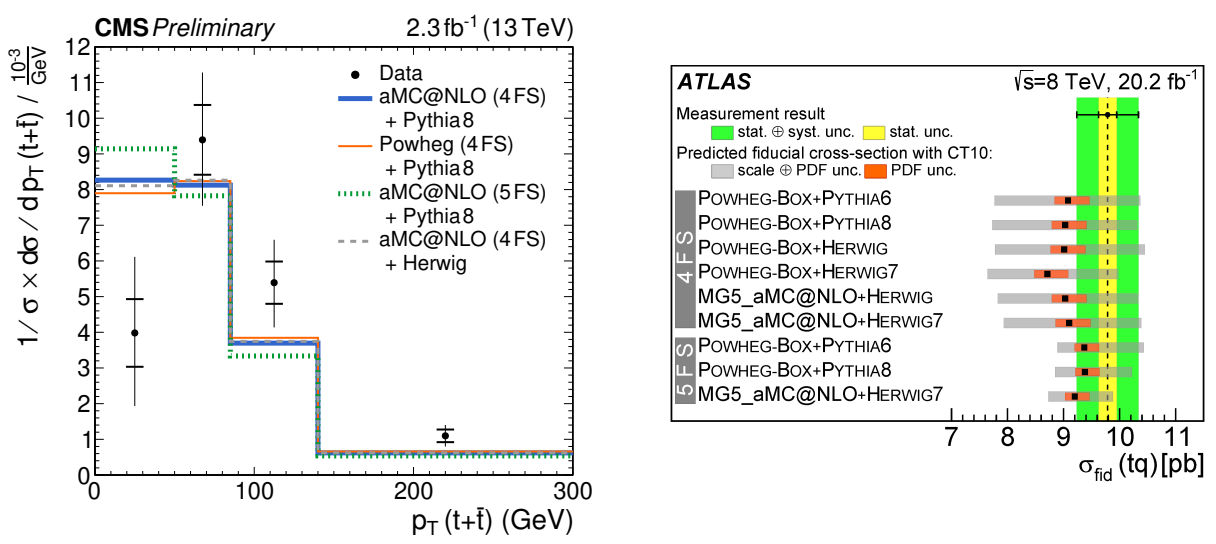

Fig. 13. Single top cross sections of the $t$-channel process $(t(\bar{t}) q$ final state). (left) differential cross section of $t$-channel single top quark production as a function of the top quark transverse momentum ${ }^{26}$. (right) Measured $t$-channel single-top fiducial cross section compared to predictions by the NLO MC generators ${ }^{27}$.

channel single top production is measurements on $V-A$ top decay helicity structure. ATLAS analysis ${ }^{28}$ selected events by simple cut-based method and achieved signalto-background ratio of about 1:1. This gave comparable result to the multi-variate analysis ${ }^{29}$ : both analyses give upper limit of better than $10 \%$ on tensor coupling normalised by the corresponding vector coupling.

The first evidence of $t Z q$ production in $p p$ collisions, an associated $Z^{0}$ production in single-top events, is reported ${ }^{30}$ recently. The diagrams for $t Z q$ production is not only sensitive to the top coupling to $Z^{0}$ but also containing diagrams with a $W W Z$ vertex (see Fig. 14 upper left and right). This process, therefore, allows to test the EW sector in the standard model. The cross section was measured by selecting events with three leptons and two jets, one of which being from the top decay and $b$-tagged, and additional jet scattered against the top quark system through $W$ exchange. A neural-network-based analysis has shown significant excess in the signal region (Fig. 14 bottom left).

The process without any jet in the final state apart from those from a top quark and a $Z^{0}$ is, as indicated in Fig. 14(bottom right), sensitive to the flavour-changing neutral current couplings since the top quark may be produced by flavour excitation through a $Z^{0}$ boson. The analysis from $\mathrm{CMS}^{31}$ gave best upper limit from the LHC, $\operatorname{Br}(t \rightarrow Z u)<0.022 \%$ and $\operatorname{Br}(t \rightarrow Z c)<0.049 \%$ at $95 \% \mathrm{CL}$.

\section{Summary}

Top quark measurements are now in the phase for precision thanks to the high statistics of LHC collisions and mature detectors there. The mass is obtained with the uncertainty of better than $0.5 \mathrm{GeV}$. Other top properties are also measured with precision. In these measurements, the dominant source of the systematic sources is 

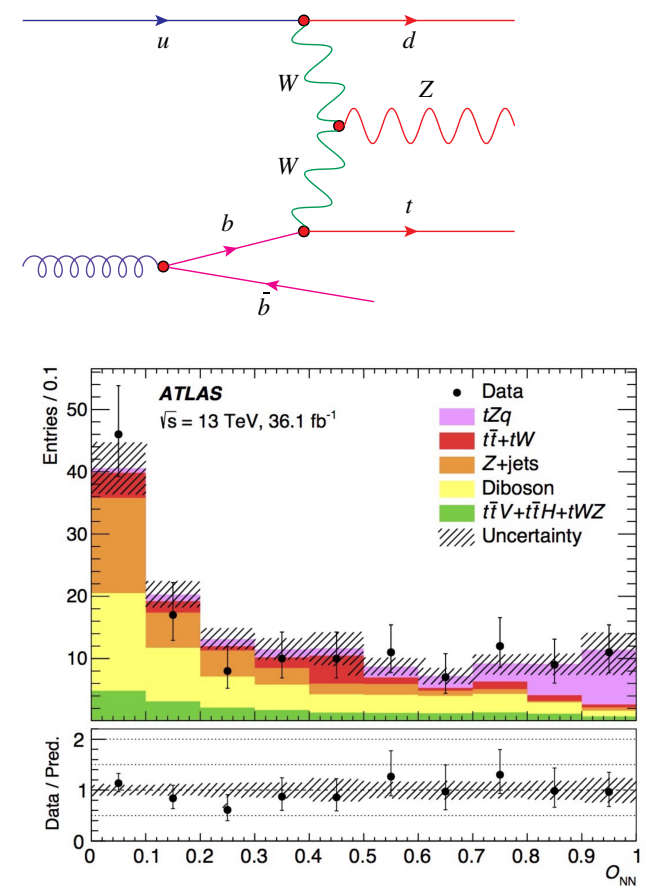
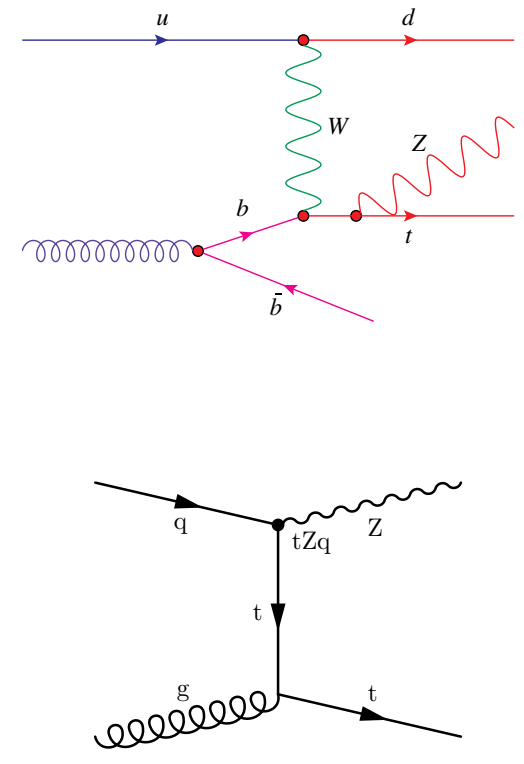

Fig. 14. (top) Two examples of $t Z q$ production in $p p$ collisions. (bottom left) The output of the neural network-based multi-variate analysis for $t Z q$ production ${ }^{30}$. (bottom right) An example of the $t Z$ final state where the top quark is produced through flavour excitation via $Z^{0}$ radiation.

not only the detector understanding but also theoretical origin. The experiments should give feedback through cross section measurements for further improving QCD model description of the top-quark production.

New processes, such as $t \bar{t} Z, t Z q$ etc., are observed recently, again thanks to the large data sets. This opens up possibility to precisely determine the EW couplings of top and weak bosons as well as possible new physics in these vertices.

The LHC is planning to obtain about three times more luminosity by the end of 2018 than those used for the data analysis presented in this symposium. This opens up further improvement on precision regime, provides more statistics for rare processes for electroweak and BSM coupling studies and increases discovery potential for new physics in high $p_{T}$ regime.

\section{References}

1. ATLAS Collaboration, G. Aad et al., The ATLAS Experiment at the CERN Large Hadron Collider, JINST 3, p. S08003 (2008).

2. CMS Collaboration, S. Chatrchyan et al., The CMS Experiment at the CERN LHC, JINST 3, p. S08004 (2008). 
3. LHCb Collaboration, A. A. Alves, Jr. et al., The LHCb Detector at the LHC, JINST 3, p. S08005 (2008).

4. CMS Collaboration, V. Khachatryan et al., Measurement of the top quark mass using proton-proton data at $\sqrt{s}=7$ and $8 \mathrm{TeV}$, Phys. Rev. D93, p. 072004 (2016).

5. LHC Top Working Group, Summary plot top mass measurements, May 2017. Available under: https://twiki.cern.ch/twiki/bin/view/LHCPhysics/TopMassHistory.

6. D0 Collaboration, B. Abbott et al., Direct measurement of the top quark mass at D0, Phys. Rev. D58, p. 052001 (1998).

7. CMS Collaboration, V. Khachatryan et al., Measurement of the mass of the top quark in decays with a $J / \psi$ meson in $p p$ collisions at $8 \mathrm{TeV}$, JHEP 12, p. 123 (2016).

8. ATLAS Collaboration, M. Aaboud et al., Measurement of lepton differential distributions and the top quark mass in $t \bar{t}$ production in $p p$ collisions at $\sqrt{s}=8 \mathrm{TeV}$ with the ATLAS detector (2017).

9. CMS Collaboration, V. Khachatryan et al., Measurement of the $t \bar{t}$ production cross section in the $e \mu$ channel in proton-proton collisions at $\sqrt{s}=7$ and $8 \mathrm{TeV}, J H E P$ 08, p. 029 (2016).

10. ATLAS Collaboration, Differential top pair cross-sections in the all-hadronic channel at $13 \mathrm{TeV}$, ATLAS-CONF-2016-100, http://cds.cern.ch/record/2217231 .

11. CMS Collaboration, Measurement of the differential cross sections of top quark pair production as a function of kinematic event variables in $p p$ collisions at $\sqrt{s}=13 \mathrm{TeV}$, CMS-PAS-TOP-16-014, http://cds.cern.ch/record/2273459 .

12. ATLAS Collaboration, M. Aaboud et al., Measurements of top-quark pair differential cross-sections in the lepton+jets channel in $p p$ collisions at $\sqrt{s}=13 \mathrm{TeV}$ using the ATLAS detector (2017).

13. ATLAS Collaboration, M. Aaboud et al., Measurements of top-quark pair differential cross-sections in the $e \mu$ channel in $p p$ collisions at $\sqrt{s}=13 \mathrm{TeV}$ using the ATLAS detector, Eur. Phys. J. C77, p. 292 (2017).

14. CMS Collaboration, V. Khachatryan et al., Measurement of differential cross sections for top quark pair production using the lepton+jets final state in proton-proton collisions at $13 \mathrm{TeV}$, Phys. Rev. D95, p. 092001 (2017).

15. CMS Collaboration, A. M. Sirunyan et al., Measurement of normalized differential $t \bar{t}$ cross sections in the dilepton channel from pp collisions at $\sqrt{s}=13 \mathrm{TeV}$ (2017).

16. M. Czakon, D. Heymes and A. Mitov, High-precision differential predictions for topquark pairs at the LHC, Phys. Rev. Lett. 116, p. 082003 (2016).

17. CMS Collaboration, A. M. Sirunyan et al., Measurements of ttbar cross sections in association with $\mathrm{b}$ jets and inclusive jets and their ratio using dilepton final states in pp collisions at sqrt(s) $=13 \mathrm{TeV}$ (2017).

18. ATLAS Collaboration, M. Aaboud et al., Measurement of jet activity produced in top-quark events with an electron, a muon and two $b$-tagged jets in the final state in $p p$ collisions at $\sqrt{s}=13 \mathrm{TeV}$ with the ATLAS detector, Eur. Phys. J. C77, p. 220 (2017).

19. LHCb Collaboration, R. Aaij et al., First observation of top quark production in the forward region, Phys. Rev. Lett. 115, p. 112001 (2015).

20. CMS Collaboration, A. M. Sirunyan et al., Measurement of the cross section for top quark pair production in association with a $\mathrm{W}$ or $\mathrm{Z}$ boson in proton-proton collisions at $\sqrt{s}=13 \mathrm{TeV}(2017)$.

21. ATLAS Collaboration, M. Aaboud et al., Measurement of the $t \bar{t} \gamma$ production cross section in proton-proton collisions at $\sqrt{s}=8 \mathrm{TeV}$ with the ATLAS detector (2017).

22. CMS Collaboration, A. M. Sirunyan et al., Measurement of the semileptonic t $\overline{\mathrm{t}}+\gamma$ production cross section in $p p$ collisions at $\sqrt{s}=8 \mathrm{TeV}$, JHEP 10, p. 006 (2017). 
23. ATLAS Collaboration, M. Aaboud et al., Direct top-quark decay width measurement in the $t \bar{t}$ lepton+jets channel at $\sqrt{s}=8 \mathrm{TeV}$ with the ATLAS experiment (2017).

24. CMS Collaboration, Bounding the top quark width using final states with two charged leptons and two jets at $\sqrt{s}=13 \mathrm{TeV}$, CMS-PAS-TOP-16-019, http://cds.cern.ch/record/2218019 .

25. LHC Top Working Group, Single Top summary of all channels at 7, 8 and $13 \mathrm{TeV}$, May 2017. Available under:

https://twiki.cern.ch/twiki/bin/view/LHCPhysics/SingleTopAllChannelsHistory.

26. CMS Collaboration, Measurement of the differential cross section for $t$ channel single-top-quark production at $\sqrt{s}=13 \mathrm{TeV}$, CMS-PAS-TOP-16-004, http://cds.cern.ch/record/2151074 .

27. ATLAS Collaboration, M. Aaboud et al., Fiducial, total and differential cross-section measurements of $t$-channel single top-quark production in $p p$ collisions at $8 \mathrm{TeV}$ using data collected by the ATLAS detector, Eur. Phys. J. C77, p. 531 (2017).

28. ATLAS Collaboration, M. Aaboud et al., Analysis of the $W t b$ vertex from the measurement of triple-differential angular decay rates of single top quarks produced in the $t$-channel at $\sqrt{s}=8 \mathrm{TeV}$ with the ATLAS detector (2017).

29. CMS Collaboration, V. Khachatryan et al., Search for anomalous $W t b$ couplings and flavour-changing neutral currents in t-channel single top quark production in $p p$ collisions at $\sqrt{s}=7$ and $8 \mathrm{TeV}$, JHEP 02, p. 028 (2017).

30. ATLAS Collaboration, M. Aaboud et al., Measurement of the production cross-section of a single top quark in association with a $Z$ boson in proton-proton collisions at 13 $\mathrm{TeV}$ with the ATLAS detector (2017).

31. CMS Collaboration, A. M. Sirunyan et al., Search for associated production of a $Z$ boson with a single top quark and for $t Z$ flavour-changing interactions in $p p$ collisions at $\sqrt{s}=8 \mathrm{TeV}$, JHEP 07, p. 003 (2017). 